\title{
Comment on "Low-frequency sea level variation and its correlation with climate events in the Pacific"
}

\author{
PARKER Albert \\ University of Ballarat, PO Box 663, Ballarat VIC 3353, Australia \\ Received September 28, 2012; accepted November 30, 2012; published online March 22, 2013
}

\begin{abstract}
The tide gauge results exhibit strong multi-decadal oscillations with detected periodicities up to a quasi-60 years. This translates in the requirement of at least 60-70 years of continuously recorded data without any quality issue and the use of a procedure appropriate for time series with periodic components to infer the acceleration trend. This is not what is done in many recent papers where the oscillating behaviour is neglected and short or incomplete records arbitrarily extended or reconstructed are used to infer wrong accelerating trends. All the tide gauges of enough length and quality show the sea levels are oscillating with important multi-decadal periodicities but absolutely not positively accelerating. The satellite radar altimeter reconstruction of the global mean sea level only available since 1993 shows despite the many uncertainties of a still imperfect procedure a not positively accelerating behaviour. The presence of acceleration in the reconstructed or extended data sets and the lack of acceleration in all the individual tide gauge measurements of enough quality and length certainly deserve further discussion. This is a comment to a previously published original paper made on the basis of other previously published results and not a new original paper.
\end{abstract}

sea level acceleration, sea level multi-decadal oscillations, tide gauge results, Pacific Ocean

Citation: Parker A. Comment on "Low-frequency sea level variation and its correlation with climate events in the pacific". Chin Sci Bull, 2013, 58: 1708-1713, doi: $10.1007 / \mathrm{s} 11434-013-5715-4$

The time series of global mean sea level (GMSL) variations between 1948 and 2007 are reconstructed in [1] by combining satellite altimeter measurements and tide gauge observations to compute a huge acceleration $0.010 \pm 0.009 \mathrm{~mm} / \mathrm{a}^{2}$ of GMSL rise in the second half of 20th century. The individual tide gauge results of enough length and quality show the sea levels are oscillating with important multidecadal periodicities but absolutely not positively accelerating. The satellite radar altimeter reconstruction of the global mean sea level only available since 1993 also shows despite the many uncertainties a not positively accelerating behaviour. The presence of acceleration in many reconstructions and the lack of acceleration in all the individual tide gauge measurements of enough quality and length certainly deserve further discussion.

Figures 1 and 2 present the monthly average sea levels and parabolic fitting for Sydney, NSW; Honolulu, HI; San

email: albertparker@y7mail.com
Diego, CA; San Francisco, CA; Seattle, WA; Victoria, BC; Vancouver, BC; Tofino, BC. Data are from [2]. Figure 1 presents the 2nd order polynomial fitting of all the available data, while Figure 2 presents the fitting of only the data 1948 to 2007 as considered in the commented paper. The 12 months moving averages are also superimposed to clear out the data of the oscillations of periodicity less than 1 year. The 2 nd order coefficients are small both positive and negative. The average acceleration or deceleration over the period of observation is twice this coefficient. There is more than an order of magnitude difference of the truly measured quantities and the value computed in the paper. The oscillations since 1948 have been clearly previously measured and there is no sign of any sharp positive acceleration 1948 to present that would have translated in much larger oscillations.

It is worth of mention that a 60 years' time window is the minimum length of a tide gauge result to clear out the longer term trend of the inter annual and multi-decadal oscillations 

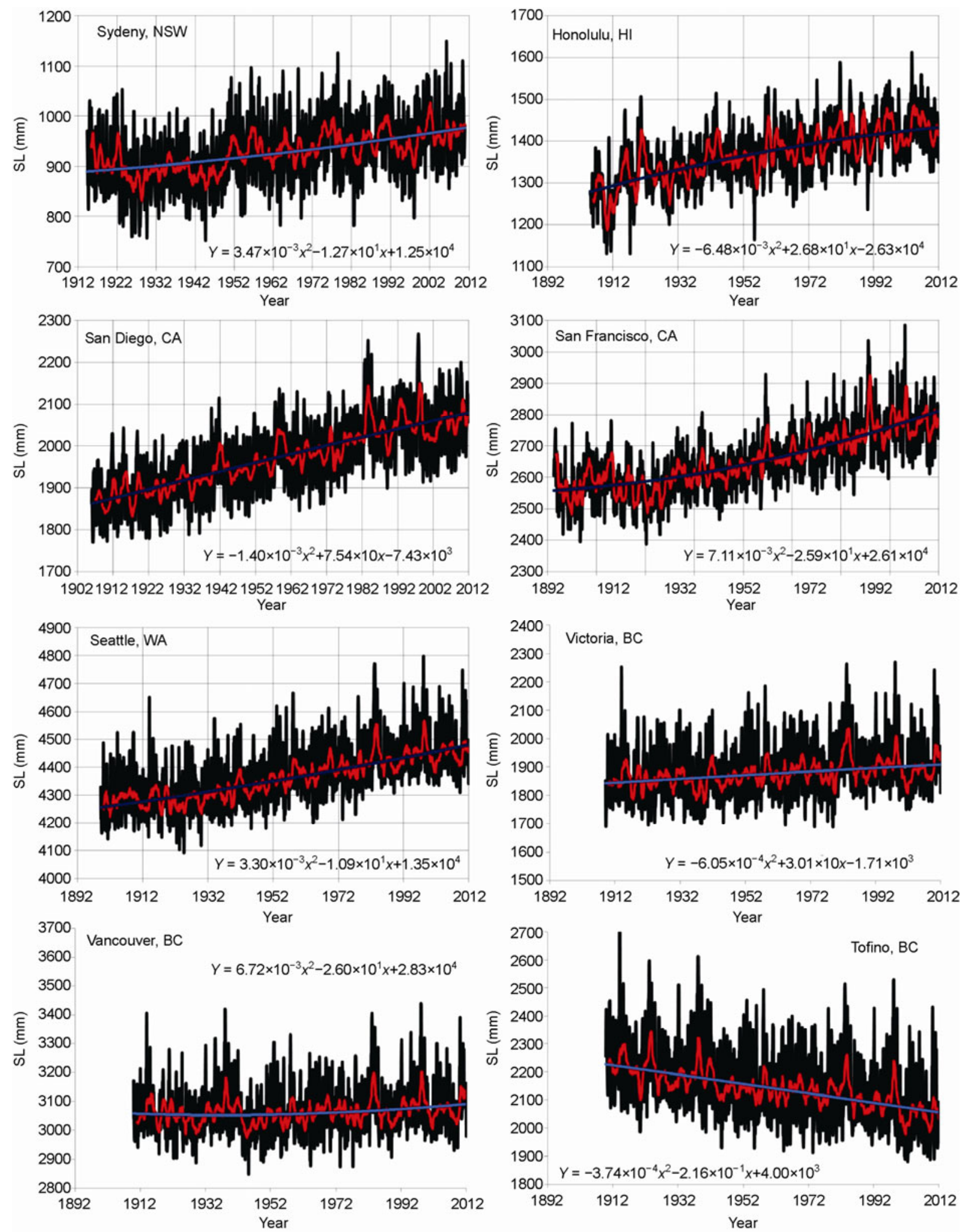

Figure 1 Monthly average sea levels and parabolic fitting for Sydney, NSW; Honolulu, HI; San Diego, CA; San Francisco, CA; Seattle, WA; Victoria, BC; Vancouver, BC; Tofino, BC. Data from PSMSL, 2012. The 2nd order coefficients are small both positive and negative. 


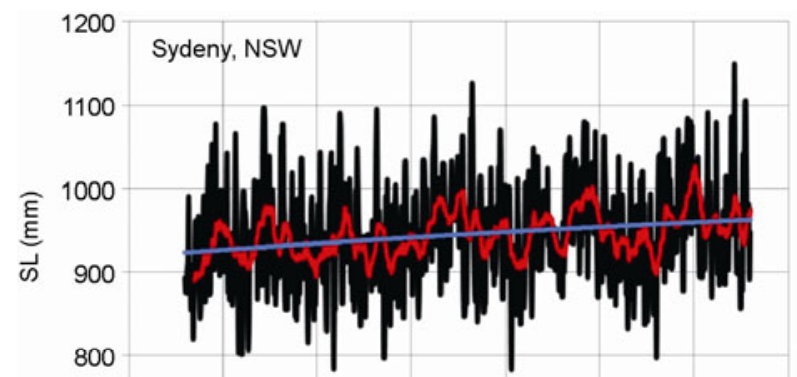

700

$Y=-2.52 \times 10^{-3} x^{2}+1.06 \times 10^{1} x-1.02 \times 10^{4}$

19121922193219421952196219721982199220022012 Year

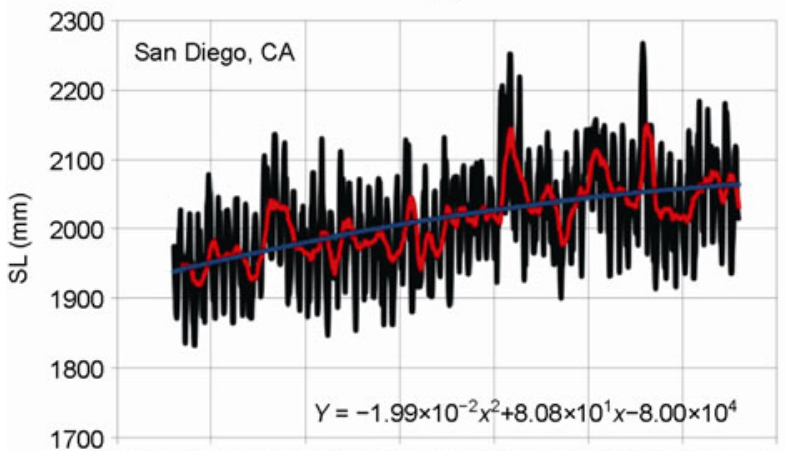

190219121922193219421952196219721982199220022012 Year
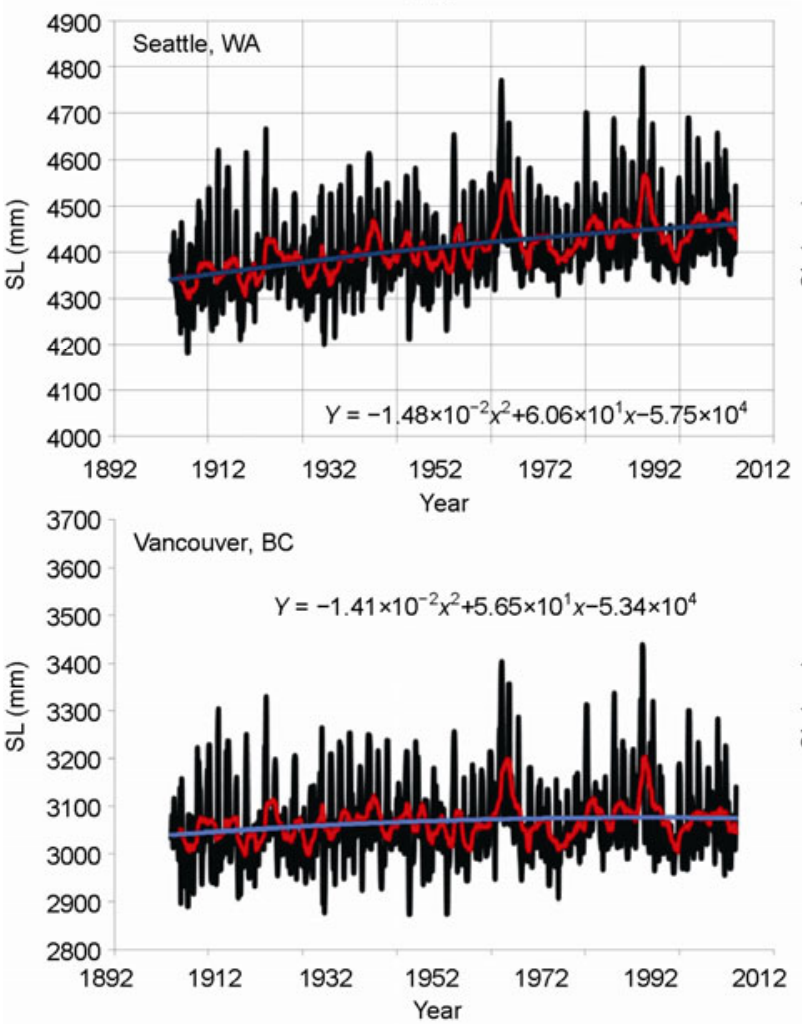
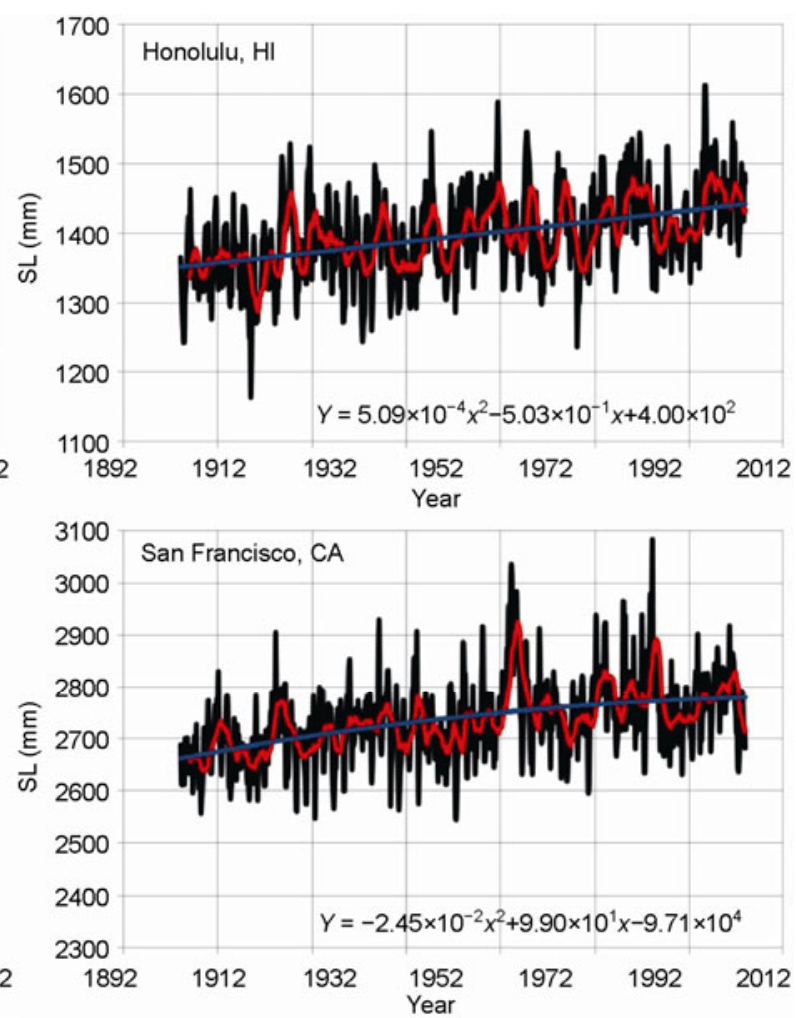

2400

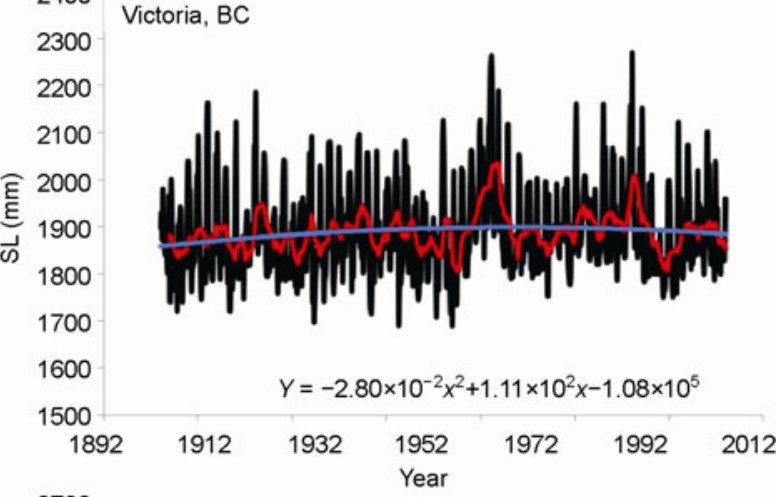

2700 Tofino, BC

2500

ิ 2400

๘ 2300

2200

2100

2000

1900

1800

1892 tonolulu, HI

Figure 2 Monthly average sea levels and parabolic fitting for Sydney, NSW; Honolulu, HI; San Diego, CA; San Francisco, CA; Seattle, WA; Victoria, BC; Vancouver, BC; Tofino, BC. Data from PSMSL, 2012. Time window of data is now 1948 to 2007 as in the commented paper. The 2nd order coefficients are small both positive and negative. 

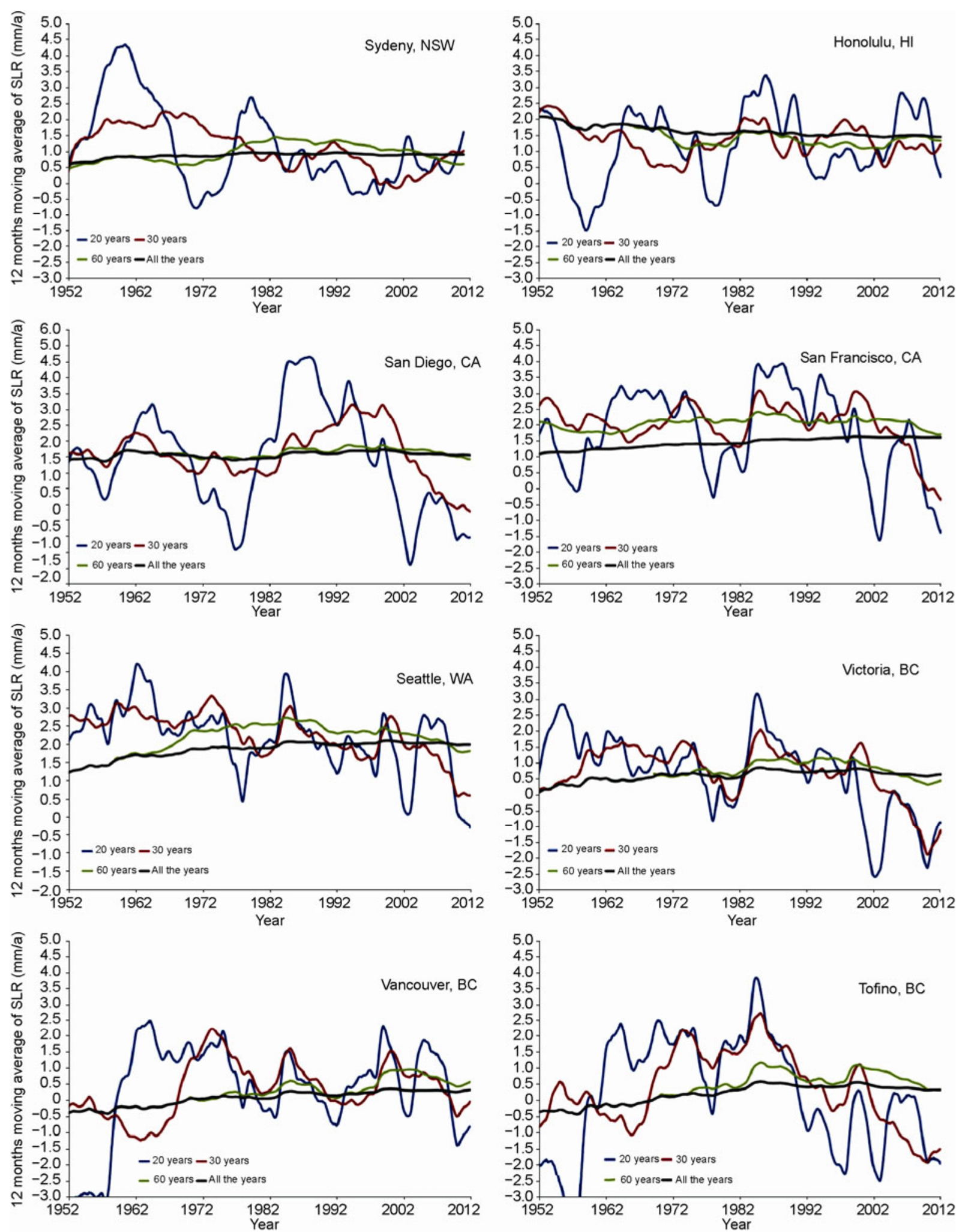

Figure 3 Computed sea level rises (SLR) by linear fitting of 20, 30, 60 years or all the data for Sydney NSW; Honolulu HI; San Francisco, CA; San Diego, CA; Seattle, WA; Victoria, BC; Vancouver, BC; Tofino, BC. Data from PSMSL, 2012. Short time windows return oscillating values. Time windows larger than 60 years return sea level rises oscillating about the longer term value. 

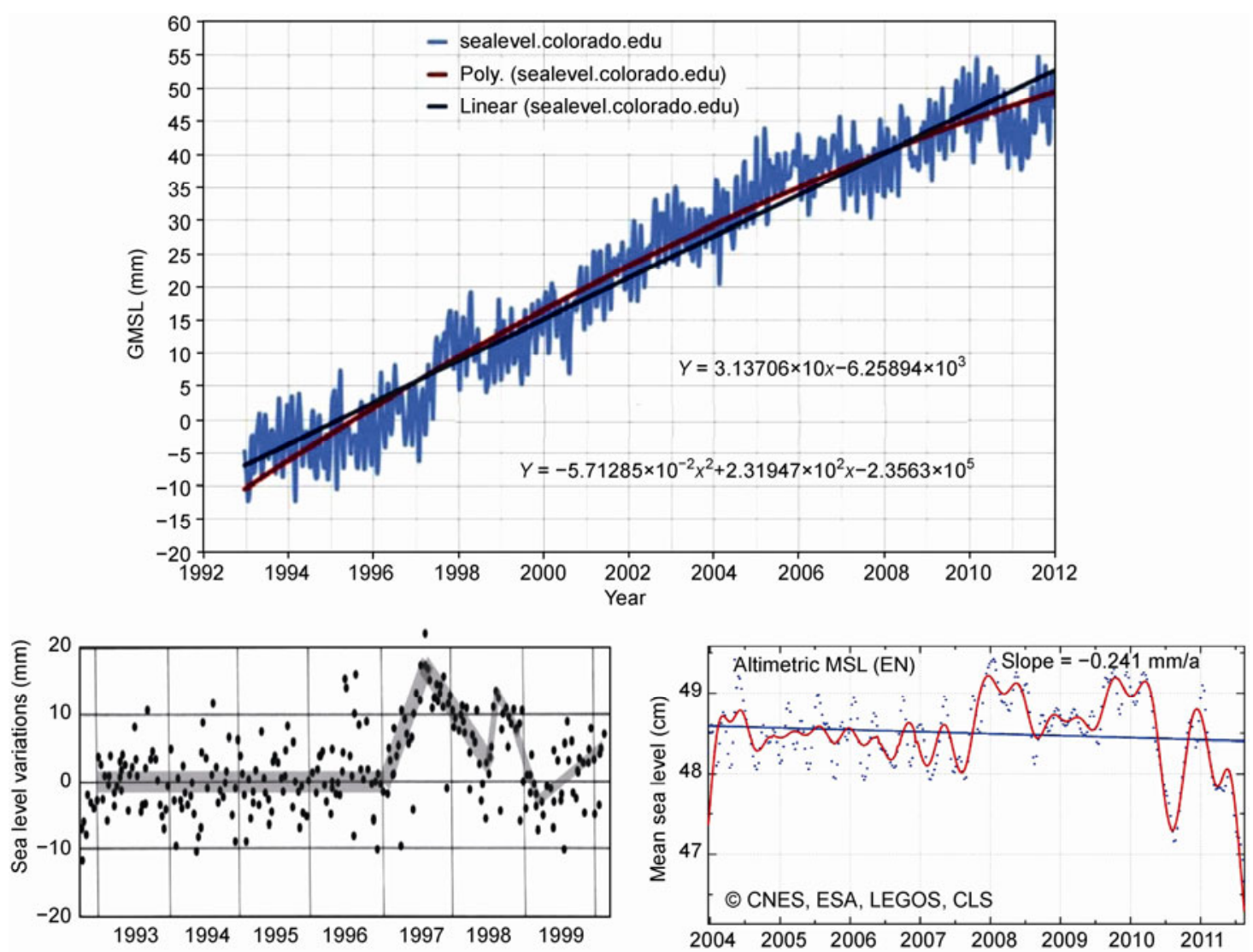

Figure 4 Top: Global mean sea level reconstruction based on satellite radar altimeters (data from University of Colorado sea level research group, 2012). The parabolic fitting has a negative 2nd order coefficient. Left: TOPEX/POSEIDON raw data before any filtering or sliding mean average October 1992 to April 2000 [12]. Right: Sea levels computed from the Envisat signal since 2004 before and after the April 2012 adjustment [13].

up to the known quasi-60 years periodicity [3-12]. Smaller time windows may return very large or small velocities and accelerations as shown by [6].

Figure 3 presents the computed sea level rises (SLR) by linear fitting of 20,30,60 years or all the data for Sydney NSW; Honolulu HI; San Francisco, CA; San Diego, CA; Seattle, WA; Victoria, BC; Vancouver, BC; Tofino, BC. In general, the $\mathrm{SLR}_{20}$ and $\mathrm{SLR}_{30}$ have large oscillations while the $\mathrm{SLR}_{60}$ has these fluctuations significantly reduced. Over the last 60 years, the $\mathrm{SLR}_{20}, \mathrm{SLR}_{30}$ and $\mathrm{SLR}_{60}$ have been moving up and down without a positive acceleration trend. The present values have been previously recorded and exceeded in all the stations. The 12 months moving averages oscillate regularly about the linear trends. The $\mathrm{SLR}_{\mathrm{A}}$ generally approaches the final long term value after $65-70$ years as in Sydney and Honolulu but sometimes it requires much more than the 65-70 years as in San Francisco where the $\mathrm{SLR}_{\mathrm{A}}$ is still changing significantly after $150 \mathrm{a}$. In all the stations, the $\mathrm{SLR}_{60}$ is not approaching the maximum value at the present time and is regularly oscillating over the last 6 decades.

Figures 1-3 consistently indicate that the measured sea levels from high quality, long recording tide gauges are oscillating and not accelerating. The reconstructed sea levels in theory based on the same results should return the same trends. The presence of acceleration in many reconstructions and the lack of acceleration in all the individual tide gauge measurements of enough quality and length certainly deserve further discussion.

Further discussion is provided in [6].

Figure 4 (top) finally presents the global mean sea level (GMSL) reconstruction based on satellite radar altimeters. Data are from [13]. The GMSL is theoretically less affected by the oscillations, and within the limits of accuracy of the computation, certainly requiring further mathematical development and more validation/calibration vs. the high quality tide gauge results, this result does not support any positive acceleration claim having the parabolic fitting a negative 2 nd order coefficient. The average acceleration or deceleration over the period of observation is twice this coefficient. This result is only available since 1993 and it is not free of criticism similarly to all the other reconstructions. Which is the contribution of what is actually measured by the satellite to the reconstructed trend is difficult to be understood, because the raw satellite altimetry data are usually not provided. Figure 4 also presents (left) the TOPEX/ POSEIDON raw data before any filtering or sliding mean average 1992 to 2000 (from [14]) and (right) the sea levels 
computed from the ENVISAT signal 2004 to 2011 before the 2012 correction of the adjustment (from [15]). These completely different behaviors also certainly deserve further discussion.

1 Jin T Y, Li J C, Jiang W P, et al. Low-frequency sea level variation and its correlation with climate events in the Pacific. Chin Sci Bull, 2012, 57: 3623-3630

2 Permanent Service on Mean Sea Level PSMSL. Sea level data. 2012, www.psmsl.org

3 National Oceanic and Atmospheric Administration NOAA, 2012, Teleconnections

4 Parker A. Sea level trends at locations of the United States with more than 100 years of recording. Nat Hazards, 2013, 65: 1011-1021

5 Parker A. Oscillations of sea level rise along the Atlantic coast of North America north of Cape Hatteras. Nat Hazards, 2013, 65: 991-997

6 Parker A. Natural oscillations and trends in long-term tide gauge records from the Pacific. Pattern Recog Phys, 2013 (in press)

7 Mazzarella A, Giuliacci A, Scafetta N. Quantifying the Multivariate ENSO Index (MEI) coupling to $\mathrm{CO}_{2}$ concentration and to the length of day variations. Theor Appl Climatol, 2012, doi: 10.1007/s00704012-0696-9

8 Scafetta N. Does the Sun work as a nuclear fusion amplifier of planetary tidal forcing? A proposal for a physical mechanism based on the mass-luminosity relation. J Atmos Solar-Terr Phys, 2012, 81-82: 2740

9 Scafetta N. Multi-scale harmonic model for solar and climate cyclical variation throughout the Holocene based on Jupiter-Saturn tidal frequencies plus the 11-year solar dynamo cycle. J Atmos Solar-Terr Phys, 2012, 80: 296-311

10 Scafetta N. Testing an astronomically based decadal-scale empirical harmonic climate model versus the IPCC (2007) general circulation climate models. J Atmos Solar-Terr Phys, 2012, 80: 124-137

11 Mazzarella A, Scafetta N. Evidences for a quasi-60-year North Atlantic Oscillation since 1700 and its meaning for global climate change. Theor Appl Climatol, 2012, 107: 599-609

12 Chambers D, Merrifield M, Nerem R. Is there a 60-year oscillation in global mean sea level? Geophys Res Lett, 2012, 39: L18607

13 University of Colorado Sea Level Research Group, GMSL. 2012, sealevel. colorado.edu/content/global-mean-sea-level-time-series-seasonalsignals- removed

14 Morner N A. Estimating future sea level changes from past records. Glob Planet Change, 2004, 40: 49-54

15 Watts A. Envisat's satellite failure launches mysteries. 2012, wattsupwiththat.com/2012/04/12/envisats-satellite-failure-launches-mysteries/

Open Access This article is distributed under the terms of the Creative Commons Attribution License which permits any use, distribution, and reproduction in any medium, provided the original author(s) and source are credited. 